PAPER

\title{
Application of simultaneous equations method to sound field creation system
}

\author{
Tadashi Ujino ${ }^{1, *}$, Keiichiroh Tanaka ${ }^{1, \dagger}$, Isao Wakabayashi, ${ }^{1, *}$, \\ Kensaku Fujii ${ }^{2, \S}$ and Mitsuji Muneyasu ${ }^{3,}$ \\ ${ }^{1}$ FUJITSU TEN LIMITED, 1-2-28 Gosho-dori, Hyogo-ku, Kobe, 652-8510 Japan \\ ${ }^{2}$ Division of Computer Engineering, University of Hyogo, \\ 2167 Shosha, Himeji, 671-2280 Japan \\ ${ }^{3}$ Faculty of Engineering Science, Kansai University, \\ 3-3-35 Yamate-cho, Suita, 564-8680 Japan
}

(Received 8 June 2009, Accepted for publication 21 April 2010)

\begin{abstract}
In this paper, we propose a sound field creation system based on the simultaneous equations method used for active noise control systems. Conventional studies on such sound field creation mainly focus on the transaural system reproducing recorded signals at the listening points. We differently purpose a system that creates the same sound field as that of a target. In the proposed system, inverse filters forming the target sound field are derived by using the simultaneous equations method, which is characterized by the use of auxiliary filters in estimating the error between the created and the target sounds. The auxiliary filters can also be used for detecting the deficiency in the number of taps of the inverse filters and in the delays to be added to the target sound field. The latter not only increases the error but also generates echoes preceding the created sound, which is annoying to listeners. In this paper, we finally verify the performance of the proposed system and also that the insufficiency can be detected by monitoring the coefficients of the auxiliary filter, by computer simulations.
\end{abstract}

Keywords: Sound field creation, Simultaneous equations method, Auxiliary filter, Inverse filter

PACS number: 43.60.Mn, 43.60.Pt [doi:10.1250/ast.31.337]

\section{INTRODUCTION}

The transaural system [1] is well known as a means of precisely reproducing a binaural recorded sound, which can be formed by canceling cross-talk between two loudspeakers and two microphones placed at the listening points. The cross-talk can be canceled by processing the recorded sound signals with inverse filters before they are provided to the loudspeakers. Moreover, the inverse filters can be derived by utilizing an adaptive filter technique [2]. The studies on the transaural system, accordingly, have resulted in the issue of the derivation of the inverse filters. Various methods have hence been proposed [3-6].

On the other hand, the acoustic paths from the loudspeakers to the microphones are assumed to be continuously changing in practical systems. Several methods of adapting to the changes, such as by compensating

\footnotetext{
*e-mail: tujino@tm.ten.fujitsu.com

†e-mail: t_ak1rou@tm.ten.fujitsu.com

‡e-mail:wakaba@tm.ten.fujitsu.com

§e-mail: fujiken@eng.u-hyogo.ac.jp

•e-mail: muneyasu@icpku.kansai-u.ac.jp
}

temperature fluctuation [7] and by applying the perturbation method or the equation error method to the derivation of the inverse filters [8,9], have hence been proposed. Each of the methods, however, has a drawback. The first method cannot deal with the change in the reverberation of the acoustic paths, the second generates extra noise, and the third involves a bias error originating in its configuration.

In this paper, we propose to apply the simultaneous equations method [10], proposed for active noise control systems, to the derivation of the inverse filters. The configuration of the active noise control system is almost the same as that of the transaural systems. Delays, inverse filters and the acoustic paths in the transaural system correspond to primary paths, noise control filters and secondary paths in the active noise control system, respectively. This means that the delays can be replaced with arbitrary acoustic paths when the simultaneous equations method is applied to the transaural system. The inverse filters can then be derived with the same procedure as that in the active noise control system. We hence extend the transaural system to a new audio system capable of creating various sound fields and providing them to 


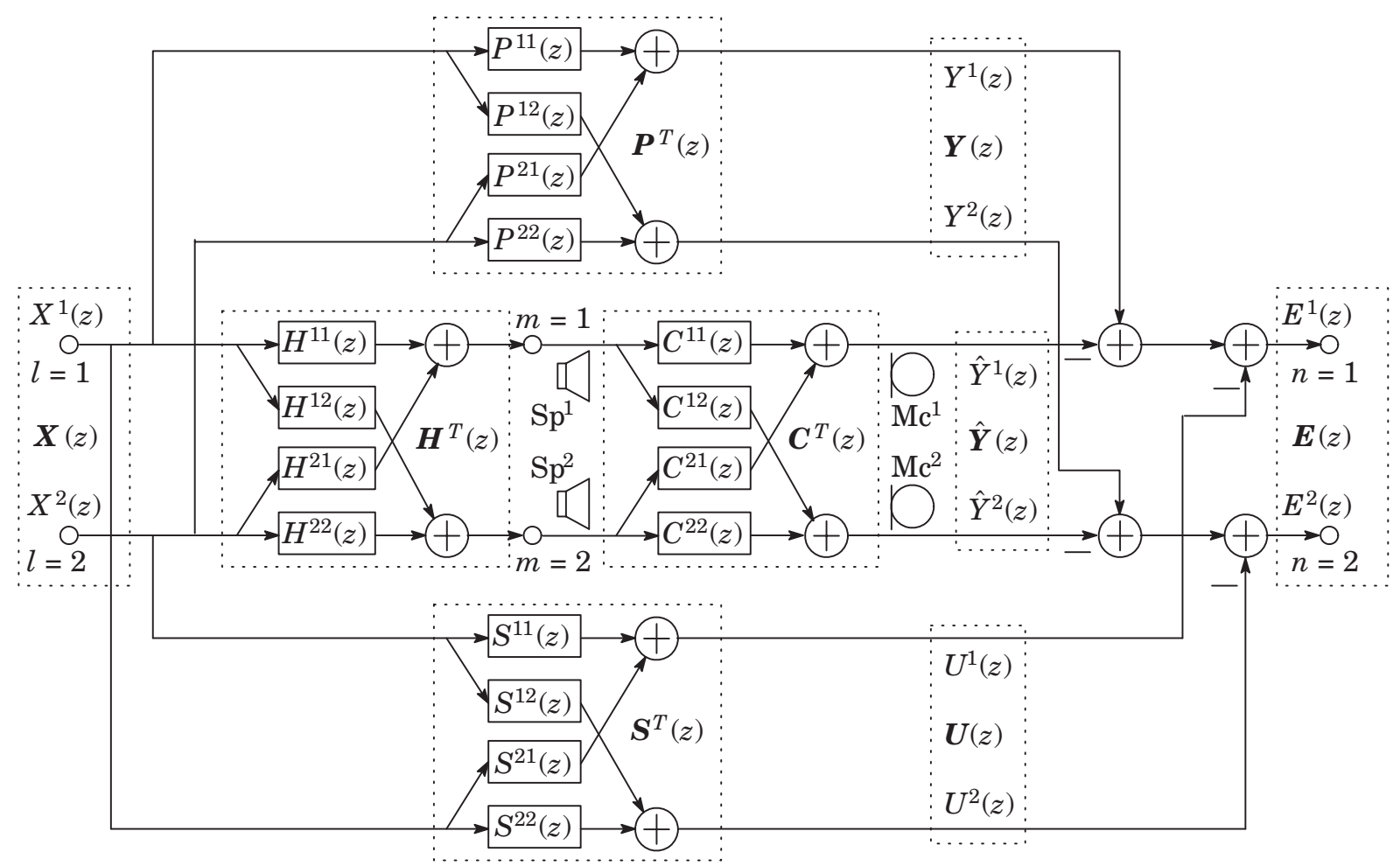

(a) Detailed block diagram

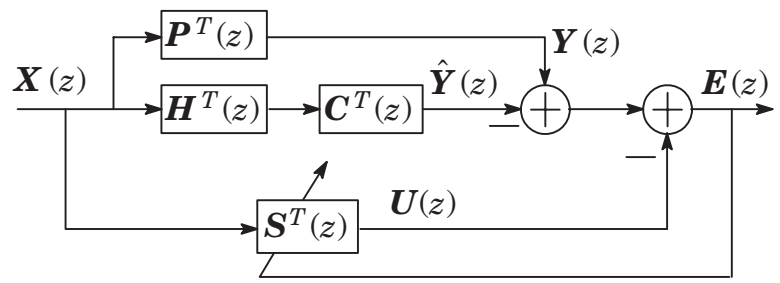

(b) Rough block diagram

Fig. 1 Configuration of proposed system.

listeners. We call this audio system a sound field creation system.

The simultaneous equations method is characterized by the use of auxiliary filters for estimating the performance of the active noise control system. When this method is applied to the sound creation system, the coefficients of the auxiliary filters indicate the lacks of the delays added to the desired responses and the number of taps of the inverse filters. By using the two impulse responses obtained from exponentially decayed normal random numbers and from practical measurement with an experimental system, we verify that the lacks can be detected from the coefficients of the auxiliary filters and that the proposed method can create arbitrary sound fields with high precision.

\section{PRINCIPLE}

Figure 1 shows the configuration of the two-channel sound field creation system proposed in this paper. The proposed system is characterized by the target sound field and the auxiliary filter matrices, $\boldsymbol{P}^{\mathrm{T}}(z)$ and $\boldsymbol{S}^{\mathrm{T}}(z)$, connected parallel to the conventional transaural system formed by the cascade connection of inverse filter matrix $\boldsymbol{H}^{\mathrm{T}}(z)$ and acoustic path matrix $\boldsymbol{C}^{\mathrm{T}}(z)$. The purpose of the proposed system is to provide the target sound to listeners at the listening points; that can be achieved when the impulse responses of the cascade connection of the inverse filters and the acoustic paths correspond to the coefficients of the target sound field filters. The proposed system creates the target sound field by utilizing the auxiliary filters identifying the overall path consisting of the target sound field filters, the inverse filters and the acoustic paths.

As seen from the configuration, this system feeds the binaural recorded sound signal vector,

$$
\boldsymbol{X}(z)=\left[X^{1}(z) X^{2}(z)\right]^{\mathrm{T}},
$$

to the inverse filter matrix,

$$
\boldsymbol{H}^{\mathrm{T}}(z)=\left[\begin{array}{l}
H^{11}(z) H^{21}(z) \\
H^{12}(z) H^{22}(z)
\end{array}\right],
$$


the target sound field filter matrix defined by a designer of this system,

$$
\boldsymbol{P}^{\mathrm{T}}(z)=\left[\begin{array}{c}
P^{11}(z) P^{21}(z) \\
P^{12}(z) P^{22}(z)
\end{array}\right],
$$

and the auxiliary filter matrix,

$$
\boldsymbol{S}(z)=\left[\begin{array}{l}
S^{11}(z) S^{21}(z) \\
S^{12}(z) S^{22}(z)
\end{array}\right] .
$$

The inverse filter matrix thereby creates the sound vector,

$$
\hat{\boldsymbol{Y}}(z)=\boldsymbol{C}^{\mathrm{T}}(z) \boldsymbol{H}^{\mathrm{T}}(z) \boldsymbol{X}(z),
$$

through the acoustic path matrix,

$$
C(z)=\left[\begin{array}{l}
C^{11}(z) C^{12}(z) \\
C^{21}(z) C^{22}(z)
\end{array}\right],
$$

from two loudspeakers $\mathrm{Sp}^{1}$ and $\mathrm{Sp}^{2}$ to two microphones $\mathrm{Mc}^{1}$ and $\mathrm{Mc}^{2}$ placed at the listening points. At the same time, the target sound field filter matrix yields the target sound vector,

$$
\boldsymbol{Y}(z)=\boldsymbol{P}^{\mathrm{T}}(z) \boldsymbol{X}(z)
$$

The auxiliary filter matrix characterizing the proposed system is used for deriving the optimum inverse filter matrix, $\boldsymbol{H}_{\mathrm{opt}}(z)$, satisfying the relation,

$$
\hat{\boldsymbol{Y}}(z)=\boldsymbol{Y}(z),
$$

which indicates

$$
\boldsymbol{P}(z)=\boldsymbol{H}_{\mathrm{opt}}(z) \boldsymbol{C}(z)
$$

This relation, moreover, means that the optimum inverse filter matrix can be derived using

$$
\boldsymbol{H}_{\mathrm{opt}}(z)=\boldsymbol{P}(z) \boldsymbol{C}^{-1}(z) .
$$

In this paper, regularity of the matrices is assumed. In practical use, the loudspeakers are arranged so that the assumption can be satisfied.

To derive the optimum inverse filter matrix, the simultaneous equations method [10] first identifies the estimation error between the created sound vector, $\hat{\boldsymbol{Y}}(z)$, and the target sound vector, $\boldsymbol{Y}(z)$, using the auxiliary filter matrix, $\boldsymbol{S}(z)$. When a tentative inverse filter matrix, $\hat{\boldsymbol{H}}(z)$, is given to the proposed system, the auxiliary filter matrix yields the following relation,

$$
\boldsymbol{S}(z)=\boldsymbol{P}(z)-\hat{\boldsymbol{H}}(z) \boldsymbol{C}(z),
$$

when the identification has been completed (that is, $\boldsymbol{E}(z)=\mathbf{0})$. Equation (11) is derived by applying $\boldsymbol{E}(z)=\mathbf{0}$ to the identification error,

$$
\begin{aligned}
\boldsymbol{E}(z) & =\{\boldsymbol{Y}(z)-\hat{\boldsymbol{Y}}(z)\}-\boldsymbol{U}(z) \\
& =\left\{\boldsymbol{P}^{\mathrm{T}}(z)-\boldsymbol{C}^{\mathrm{T}}(z) \boldsymbol{H}^{\mathrm{T}}(z)-\boldsymbol{S}^{\mathrm{T}}(z)\right\} \boldsymbol{X}(z),
\end{aligned}
$$

where

$$
\boldsymbol{U}(z)=\boldsymbol{S}^{\mathrm{T}}(z) \boldsymbol{X}(z)=\left[U^{1}(z) U^{2}(z)\right]^{\mathrm{T}}
$$

is the output vector provided by the auxiliary filter matrix.

In active noise control systems, $\boldsymbol{P}(z)$ and $\boldsymbol{C}(z)$ are unknown. The simultaneous equations method accordingly gives two different inverse filter matrices to the active noise control system at a proper interval, and then derives $\boldsymbol{H}_{\mathrm{opt}}(z)$ by using the estimated two auxiliary filter matrices. However, $\boldsymbol{P}(z)$ defined by the designer is known in the proposed system, and only $\boldsymbol{C}(z)$ is unknown. In this case, the only unknown, $\boldsymbol{C}(z)$, can be directly derived from (11) as

$$
\boldsymbol{C}(z)=\hat{\boldsymbol{H}}^{-1}(z)\{\boldsymbol{P}(z)-\boldsymbol{S}(z)\}
$$

which, moreover, can be rewritten to

$$
\boldsymbol{C}^{-1}(z)=(\boldsymbol{P}(z)-\boldsymbol{S}(z))^{-1} \hat{\boldsymbol{H}}(z) .
$$

Substituting $\boldsymbol{C}^{-1}(z)$ into (10) finally yields

$$
\boldsymbol{H}_{\mathrm{opt}}(z)=\boldsymbol{P}(z)(\boldsymbol{P}(z)-\boldsymbol{S}(z))^{-1} \hat{\boldsymbol{H}}(z) .
$$

Incidentally, applying the simple delay matrix

$$
\boldsymbol{P}(z)=\left[\begin{array}{cc}
z^{-D} & 0 \\
0 & z^{-D}
\end{array}\right]
$$

to the proposed system forms a transaural system.

\section{TRANSFORM TO FILTER COEFFICIENT}

$\boldsymbol{H}_{\mathrm{opt}}(z)$ shown in (16) gives a transfer function matrix expressed with the $z$-transform. For applying the above principle to practical systems, the transformation of the transfer function matrix to a filter coefficient matrix is required. In this paper, we apply the frequency domain technique used in [10] to the transformation. In the frequency domain, (16) is rewritten as

$$
\boldsymbol{H}_{\mathrm{opt}}=\boldsymbol{P}(\boldsymbol{P}-\boldsymbol{S})^{-1} \hat{\boldsymbol{H}},
$$

where the elements of

$$
\begin{aligned}
& P=\left[\begin{array}{c}
P^{11} P^{12} \\
P^{21} P^{22}
\end{array}\right], \\
& S=\left[\begin{array}{c}
S^{11} S^{12} \\
S^{21} S^{22}
\end{array}\right]
\end{aligned}
$$

and

$$
\hat{\boldsymbol{H}}=\left[\begin{array}{c}
\hat{\boldsymbol{H}}^{11} \hat{\boldsymbol{H}}^{12} \\
\hat{\boldsymbol{H}}^{21} \hat{\boldsymbol{H}}^{22}
\end{array}\right]
$$

are discrete Fourier transforms (DFTs) of the coefficient vectors given to the target sound field, the auxiliary and the inverse filters, respectively.

Here, it should be noted that DFT is applied not to the coefficient vectors of target sound field filter $P^{l n}(z)$, auxiliary filter $S^{l n}(z)$ and tentative inverse filter $\hat{H}^{l m}(z)$, that is, 


$$
\begin{aligned}
\boldsymbol{p}^{l n} & =\left[p^{l n}(0) \cdots p^{l n}(I-1)\right]^{\mathrm{T}}, \\
\boldsymbol{s}^{l n} & =\left[s^{l n}(0) \cdots s^{l n}(I-1)\right]^{\mathrm{T}}
\end{aligned}
$$

and

$$
\hat{\boldsymbol{h}}^{l m}=\left[\hat{h}^{l m}(0) \cdots \hat{h}^{l m}(J-1)\right]^{\mathrm{T}},
$$

but to the modified coefficient vectors to which zeros are added,

$$
\begin{aligned}
\tilde{\boldsymbol{p}}^{l n} & =[p^{\ln }(0) \cdots p^{\ln }(I-1) \underbrace{0 \cdots 0}_{I}]^{\mathrm{T}}, \\
\tilde{\boldsymbol{s}}^{l n} & =[s^{\ln }(0) \cdots s^{\ln }(I-1) \underbrace{0 \cdots 0}_{I}]^{\mathrm{T}}
\end{aligned}
$$

and

$$
\tilde{\boldsymbol{h}}^{l m}=[\hat{h}^{l m}(0) \cdots \hat{h}^{l m}(J-1) \underbrace{0 \ldots 0}_{2 I-J}]^{\mathrm{T}},
$$

where $I$ and $J$ are the number of taps of the filters, and $l=1,2, m=1,2$ and $n=1,2$ denote the column and the row numbers of the filter matrices, respectively. Circular convolutions provided by DFT are thereby converted to linear convolutions. The coefficient vectors of the optimum inverse filter matrix can then be derived by extracting the former $J$ components of each element $\tilde{\boldsymbol{h}}^{l m}$ obtained by applying inverse discrete Fourier transform (IDFT) to $\boldsymbol{H}_{\text {opt }}$.

\section{UPDATING PROCEDURE}

In practical use, the acoustic paths should be assumed to be continuously changing. The proposed system is accordingly designed so as to estimate $\boldsymbol{H}_{\text {opt }}(z)$ repeatedly. The current coefficient vectors of the optimum inverse filters can be repeatedly estimated by executing the following procedure.

(1) Give a coefficient vector matrix, for example,

$$
\left\{\begin{array}{l}
\hat{\boldsymbol{h}}^{11}=\left[\begin{array}{lll}
a & 0 & \cdots
\end{array}\right]^{\mathrm{T}} \\
\hat{\boldsymbol{h}}^{12}=\left[\begin{array}{lll}
0 & 0 & \cdots
\end{array}\right]^{\mathrm{T}} \\
\hat{\boldsymbol{h}}^{21}=\left[\begin{array}{lll}
0 & 0 & \cdots
\end{array}\right]^{\mathrm{T}} \\
\hat{\boldsymbol{h}}^{22}=\left[\begin{array}{lll}
b & 0 & \cdots
\end{array}\right]^{\mathrm{T}}
\end{array},\right.
$$

to the inverse filter matrix, where $a$ and $b$ are arbitrary constants.

(2) Calculate DFT of $\tilde{\boldsymbol{h}}^{l m}$.

(3) Estimate $\boldsymbol{s}^{l n}$.

(4) Calculate DFT of $\boldsymbol{s}^{n}$.

(5) Calculate $\boldsymbol{H}_{\text {opt }}$ using (18).

(6) Extract former $J$ components of IDFT of $\boldsymbol{H}_{\text {opt }}$.

(7) Give extracted $\hat{\boldsymbol{h}}^{l m}$ to inverse filter matrix.

(8) Go back to (2).

By repeating the above updating procedure from (2) to (7), the proposed system can automatically create the sound

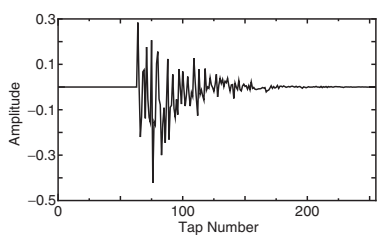

(a) $p^{11}$

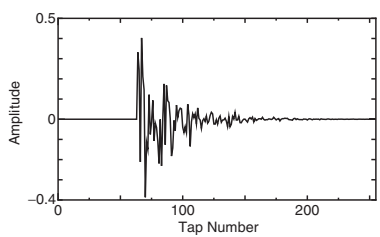

(c) $p^{21}$

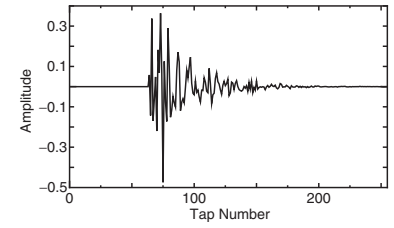

(b) $p^{12}$

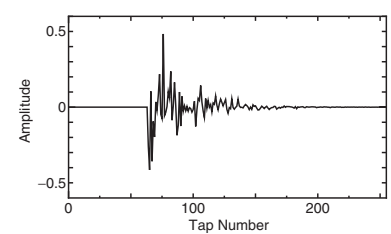

(d) $p^{22}$
Fig. 2 Coefficient vectors of target sound field filters.

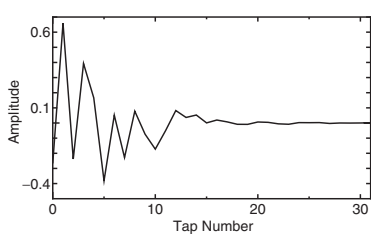

(a) $c^{11}$

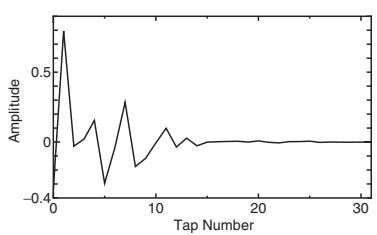

(c) $c^{21}$

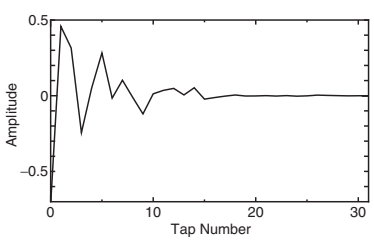

(b) $c^{12}$

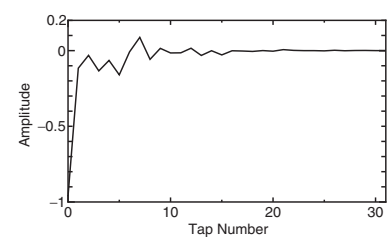

(d) $c^{22}$
Fig. 3 Impulse responses of acoustic paths.

vector approximated to the target sound vector, even if the acoustic paths change.

\section{VERIFICATION BY COMPUTER SIMULATION}

We next verify the performance of the proposed system through computer simulations. Figures 2 and 3 show the coefficient vectors of the target sound field filters and the impulse response samples of the acoustic paths, where they are given by exponentially decayed normal random numbers. In addition, the sound creation path matrix,

$$
\hat{\boldsymbol{P}}=\hat{\boldsymbol{H}} \boldsymbol{C},
$$

naturally generates some group delays. In this simulation, the group delays are tentatively estimated to be 64 sample times.

In the proposed system, various adaptive algorithms can be used for estimating the coefficient vectors of the auxiliary filters. We use the well-known algorithm, 


$$
s_{j+1}^{l n}=s_{j+1}^{l n}+\mu \frac{e_{j}^{n} x_{j}^{l}}{x_{j}^{1^{T}} x_{j}^{1}+x_{j}^{2^{T}} x_{j}^{2}},
$$

for this estimation, where $s_{j}^{l n}$ is an auxiliary filter coefficient vector given at $j$ sample time, $\boldsymbol{x}_{j}^{l}$ is a recorded sound signal vector, $\mu$ is a constant called step size, and

$$
e_{j}^{n}=\left\{y_{j}^{n}-\hat{y}_{j}^{n}\right\}-u_{j}^{n}
$$

is the difference between the output of the auxiliary filter, $u_{j}^{n}$, and the difference between the target and the created sounds, $\left\{y_{j}^{n}-\hat{y}_{j}^{n}\right\}$. Here, $\hat{y}_{j}^{n}$ is the created sound signal detected by microphone $\mathrm{Mc}^{n}$, and $y_{j}^{n}$ is the desired signal yielded by the target sound field filters. The coefficient vectors of the auxiliary filters are estimated using the above algorithm, and the coefficient vectors of the optimum inverse filters are later calculated by applying IDFT to (18).

To simplify the verification, we use

$$
x_{j}^{1}=r_{j}^{0}+r_{j}^{1}
$$

and

$$
x_{j}^{2}=r_{j}^{0}+r_{j}^{2}
$$

as the recorded sound signals, where $r_{j}^{0}, r_{j}^{1}$ and $r_{j}^{2}$ are white noise. In practical systems, the recorded sound signals are strongly correlated with each other [11]. The degree of cross-correlation can be estimated from the power ratio of $r_{j}^{0}$ to $r_{j}^{m}$. In this simulation, we assume the power ratio to be $20 \mathrm{~dB}$. In addition, the other conditions are

(1) number of taps of inverse filters, $J=224$;

(2) number of samples of acoustic paths, $K=32$;

(3) number of taps of auxiliary filters, $I=256$;

(4) number of taps of target sound field filters, $I=256$;

(5) duration of DFT, $2 I=512$;

(6) power ratio of recorded signals to environmental noise detected by microphones $\mathrm{Mc}^{1}$ and $\mathrm{Mc}^{2}$ shown in Fig. 1, $-40 \mathrm{~dB}$;

(7) step size, $\mu=1.0$;

(8) number of times of updating coefficient vectors of auxiliary filters: $2^{18}=262,144$.

Figure 4 shows the transition of the estimation errors calculated using

$$
E_{k}^{l n}=10 \log _{10} \frac{\sum_{l=1}^{2} \sum_{n=1}^{2} \sum_{i=0}^{I-1}\left\{p^{l n}(i)-\hat{p}_{k}^{l n}(i)\right\}^{2}}{\sum_{l=1}^{2} \sum_{n=1}^{2} \sum_{i=0}^{I-1}\left\{p^{l n}(i)\right\}^{2}},
$$

where, $\hat{p}_{k}^{l n}(i)$ is the $i$ th impulse response sample of element ln of the sound creation path matrix obtained by repeating the updating procedure $k$ times. The estimation error, $E_{k}^{l n}$, presents the difference between the target and the created sounds. This result shows that the proposed system can create sound approximating the target sound.

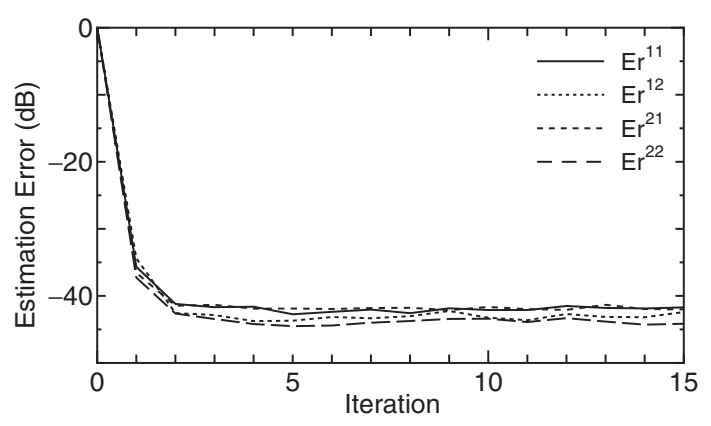

Fig. 4 Transition of estimation errors where number of taps is 224 and sample time of added delays is 64 .

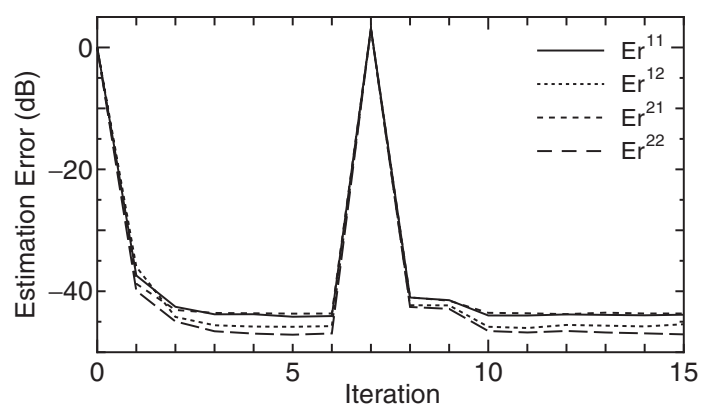

Fig. 5 Transition of estimation errors obtained by changing acoustic paths where number of taps is 224 and sample time of added delays is 64 .

Figure 5 shows the transition of the estimation errors obtained by reversing the signs $( \pm)$ of the impulse response samples of the acoustic paths just before updating the coefficient vectors of the inverse filters at $k=7$. As seen from the result, the estimation errors increase to $3 \mathrm{~dB}$ because of the path change and subsequently quickly decrease to less than $-40 \mathrm{~dB}$. This result shows that the proposed system can automatically decrease the estimation error increased by acoustic path changes.

\section{DELAY AND NUMBER OF TAPS}

The sound creation paths naturally have some group delays, which requires the addition of sufficient delays to the target sound path filters. The lack of added delays degrades the performance of the sound creation system. Figure 6 shows the transitions of the estimation errors calculated by adding the delay of 32 sample times to the target sound field filters, where the other simulation conditions are the same as those in the case of Fig. 4. In comparison with the results shown in Fig. 4, the estimation errors increase by more than $10 \mathrm{~dB}$.

In addition, Fig. 7 shows the impulse responses of the sound creation paths calculated by reducing the delays from 64 to 32 sample times. In Fig. 7, we can see some echoes preceding the substance of the created sound, which is annoying listeners. 


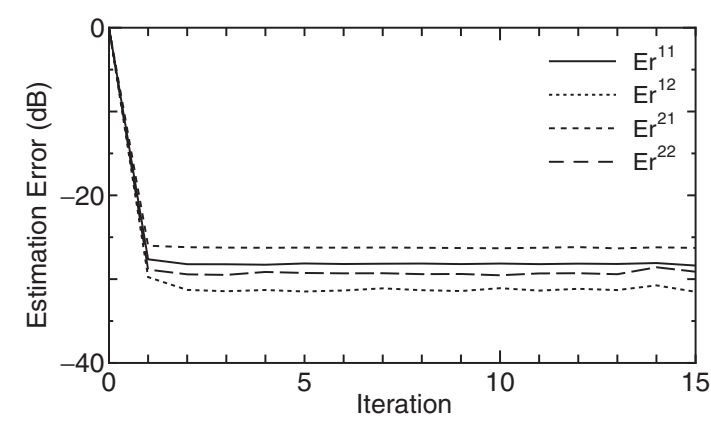

Fig. 6 Transition of estimation errors where number of taps is 224 and sample time of added delays is 32 .

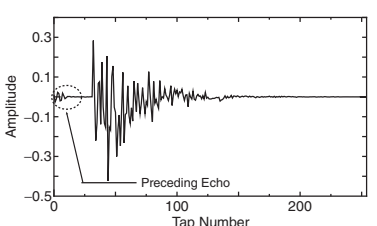

(a) $\hat{p}^{11}$

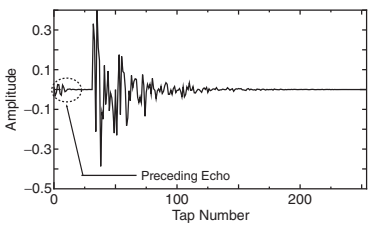

(c) $\hat{p}^{21}$

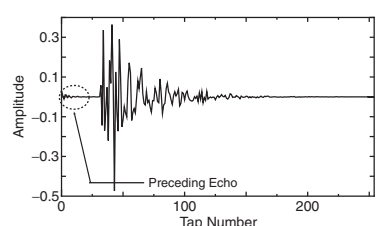

(b) $\hat{p}^{12}$

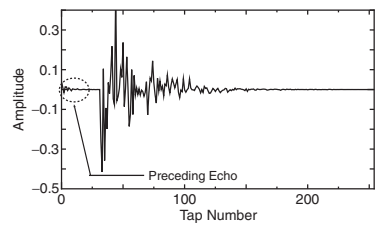

(d) $\hat{p}^{22}$
Fig. 7 Impulse responses of sound creation paths where number of taps is 224 and sample time of added delays is 32 .

An insufficient number of taps given to the inverse filters also increases the difference between the target and the created sounds. Figure 8 shows the transitions of the estimation errors calculated upon reducing the number of taps of the inverse filters from 224 to 128 . In this example, the decreases in the estimation errors are saturated at about $-20 \mathrm{~dB}$, which means the degradation of $20 \mathrm{~dB}$ in comparison with those in the case of 224 taps.

Both insufficiencies thus increase the estimation errors. To reduce the estimation errors, sound field creation systems are required to detect which type of insufficiency causes the degradation and then to properly increase the delays or the taps. In the proposed system, the coefficients of the auxiliary filters indicate which of the two is insufficient. Figures 9 and 10 show the coefficients of the auxiliary filters obtained when the added delays are 32 and 64 sample times, respectively. Clearly, the former part of the coefficients shown in Fig. 9 is about ten times that in the case of 64 sample time delays, which indicates the generation of the preceding echoes.

The insufficient number of taps given to the inverse filters can be also identified by the coefficients of the

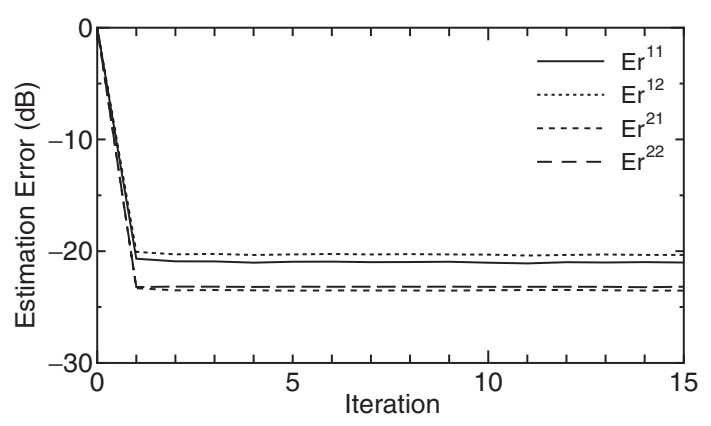

Fig. 8 Transition of estimation errors where number of taps is 128 and sample time of added delays is 64 .

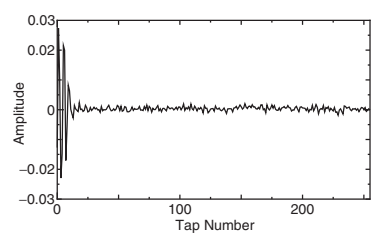

(a) $s^{11}$

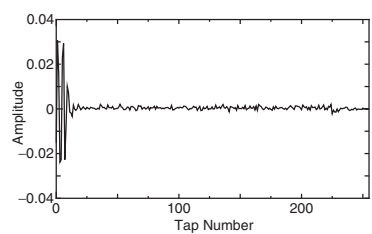

(c) $s^{21}$

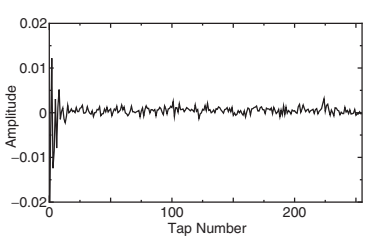

(b) $s^{12}$

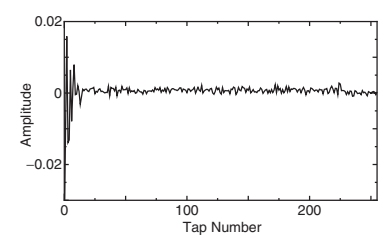

(d) $s^{22}$
Fig. 9 Coefficients of auxiliary filters where number of taps is 224 and sample time of added delays is 32 .

auxiliary filters. Figure 11 shows the coefficients of the auxiliary filters calculated upon reducing the number of taps from 224 to 128. Apparently, the amplitudes of the coefficients of the auxiliary filters in their latter parts is more than those in the case of 224 taps, which indicates the insufficient number of taps. The coefficients of the auxiliary filters thus indicate the difference in the impulse responses of the sound creation paths and the target sound fields. In the proposed system, the numbers of added delays and taps can be adjusted by monitoring the coefficients of the auxiliary filters.

\section{VERIFICATION USING PRACTICAL IMPULSE RESPONSES}

Finally, we verifies the performance of the proposed method using practical impulse responses. Figure 12 shows a torso simulator (HATS-4128C) and loudspeakers placed in an experimental acoustic room, arranged considering the application to 5.1 channel surround systems, as shown in Fig. 13. The impulse responses are measured using the loudspeakers and the microphones built in the torso simulator, and the results are presented in Fig. 14. In 


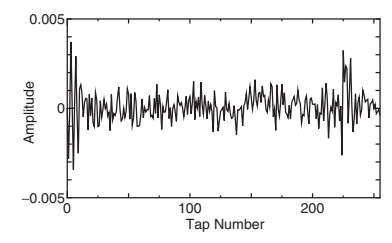

(a) $s^{11}$

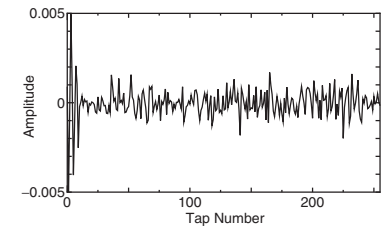

(c) $s^{21}$

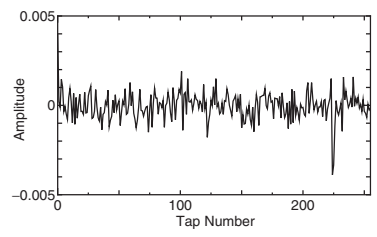

(b) $s^{12}$

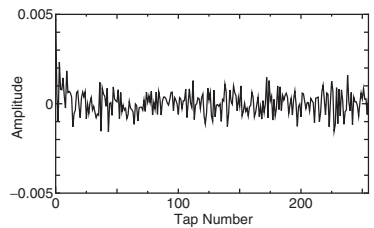

(d) $s^{22}$
Fig. 10 Coefficients of auxiliary filters where number of taps is 224 and sample time of added delays is 64 .

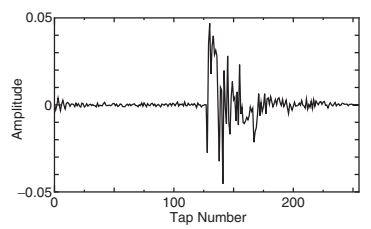

(a) $s^{11}$

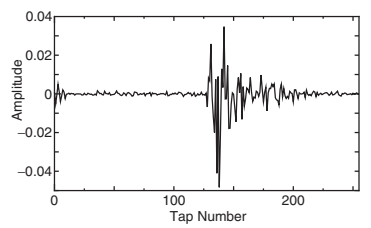

(c) $s^{21}$

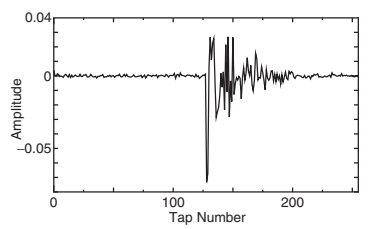

(b) $s^{12}$

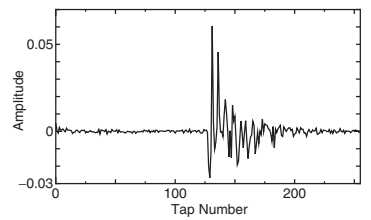

(d) $s^{22}$
Fig. 11 Coefficients of auxiliary filters where number of taps is 128 and sample time of added delays is 64 .

practical use, the microphones are placed close to the listener's ears. The equipment used for the measurement is also listed in Table 1. In addition, Fig. 15 shows the coefficients of the target sound field filters used for the verification, where the coefficients are given by exponential decayed normal random numbers, such as in Fig. 2.

Figure 16 shows the transition of the estimation errors obtained using the practical impulse responses, where the number of impulse response samples of the acoustic paths is 128 , the delays added to the impulse responses of the target sound field filters are changed from 64 given in Fig. 2 to 256 sample times, and the other conditions are

(1) number of taps of inverse filters, $J=896$;

(2) number of taps of auxiliary filters, $I=1,024$;

(3) duration of DFT, $2 I=2,048$;

(4) number of times of updating coefficients of auxiliary filters, $1,024 \times 1,024$.

As seen from the results shown in Fig. 16, the estimation errors decrease to less than $-15 \mathrm{~dB}$, which

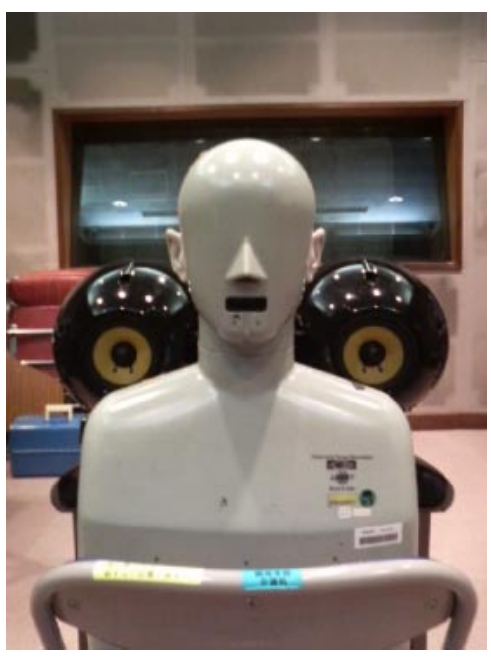

Fig. 12 Experimental system used for measuring impulse responses.

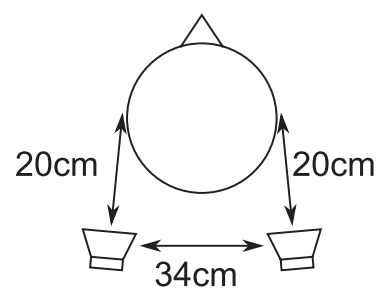

Fig. 13 Arrangement of simulator and loudspeakers used for measuring impulse responses.

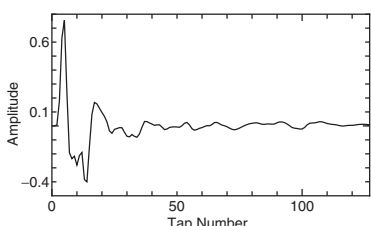

(a) $c^{11}$

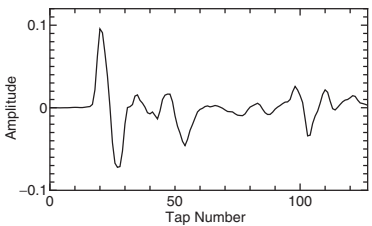

(c) $c^{21}$

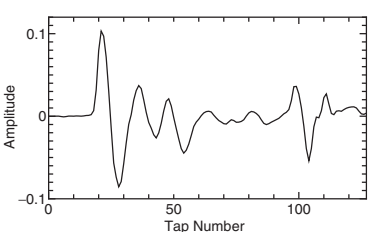

(b) $c^{12}$

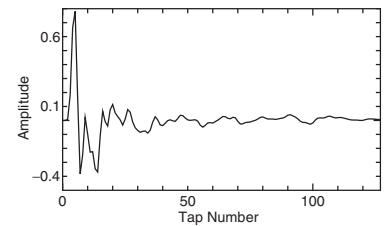

(d) $c^{22}$

Fig. 14 Impulse responses of practical acoustic paths measured in experimental room.

Table 1 Equipment used for measuring impulse responses.

Loudspeaker

Simulator

Microphone Amplifier

Power Amplifier

Audio Sound Analyzer

Audio Sound Card
FUJITSU TEN, TD307

Brüel \& Kjær, 4128C

YAMAHA, MLA7

YAMAHA, P4050

ETANI Electronics, ASA-2 M-Audio, DELTA-66 


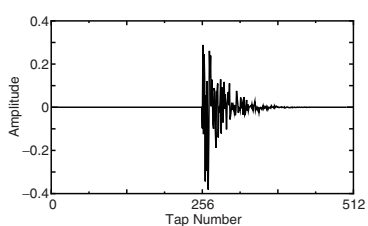

(a) $p^{11}$

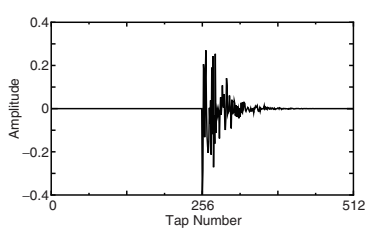

(c) $p^{21}$

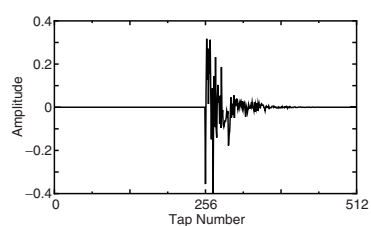

(b) $p^{12}$

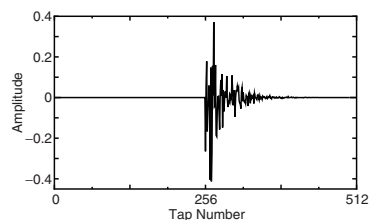

(d) $p^{22}$
Fig. 15 Coefficient vectors of target sound field filters applied to simulations using practical impulse responses.

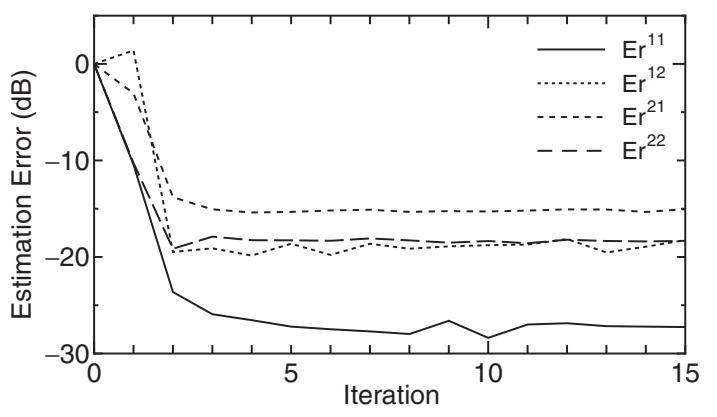

Fig. 16 Transition of estimation errors obtained using practical impulse responses $(J=896, D=256)$.

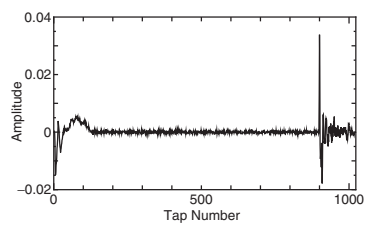

(a) $s^{11}$

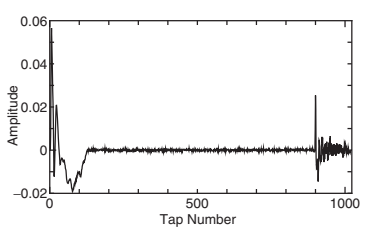

(c) $s^{21}$

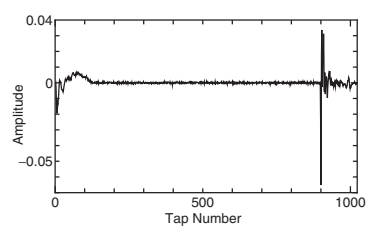

(b) $s^{12}$

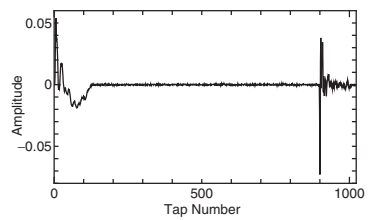

(d) $s^{22}$
Fig. 17 Coefficients of auxiliary filters $(J=896$ and $D=256)$.

demonstrates that the proposed method works well in practice. Actually, the coefficients of the auxiliary filters demonstrate that the proposed system creates a sound field that approximates to the target sound field, except for the head and the tail of the impulse responses, as shown in Fig. 17. Figure 18 shows the impulse responses of the

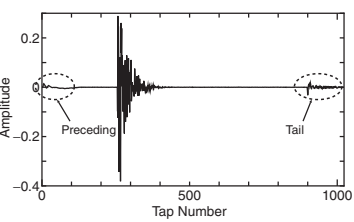

(a) $\hat{p}^{11}$

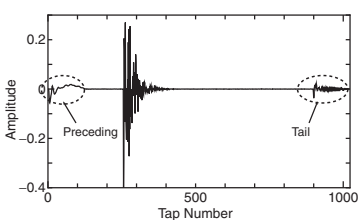

(c) $\hat{p}^{21}$

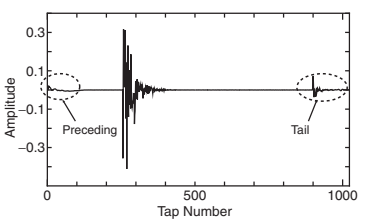

(b) $\hat{p}^{12}$

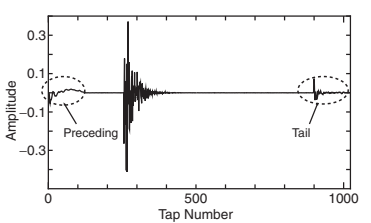

(d) $\hat{p}^{22}$
Fig. 18 Impulse responses of created sound field $(J=896$ and $D=256)$.

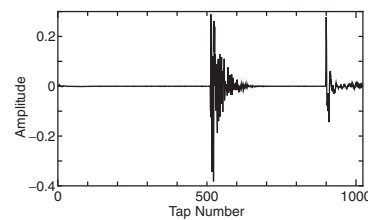

(a) $\hat{p}^{11}$

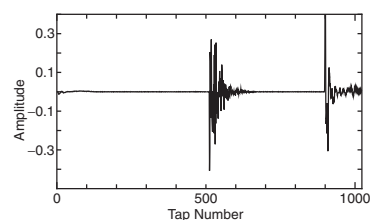

(c) $\hat{p}^{21}$

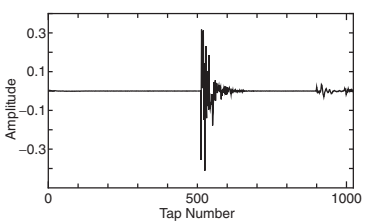

(b) $\hat{p}^{12}$

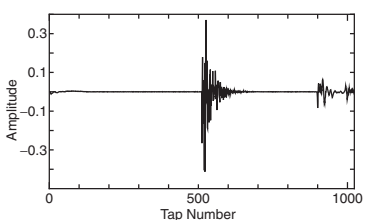

(d) $\hat{p}^{22}$
Fig. 19 Impulse responses of created sound field $(J=896$ and $D=512)$.

created sound field. As seen from this result, we can see some preceding echoes and tail echoes in the impulse responses. The tail and preceding echoes indicate the insuffficient number of taps and delays, respectively.

As mentioned above, the preceding echoes can be reduced by increasing the delays involved in the target sound field filters. Figure 19 shows the impulse responses of the created sound field obtained by increasing the delays from 256 to 512 sample times, where the number of taps of the inverse filters is held at 896 . The preceding echoes accordingly decrease; however, the tail echoes increase. This can also be recognized from the auxiliary filter coefficients shown in Fig. 20.

The tail echoes can be similarly reduced by increasing the number of taps of the inverse filters. Figure 21 shows the impulse responses of the created sound field obtained by increasing the number of taps of the inverse filters from 896 to 1280 , where the delays involved in the target sound field filter is returned to 256 sample times. The preceding echoes accordingly increase, and oppositely, the tail echoes 


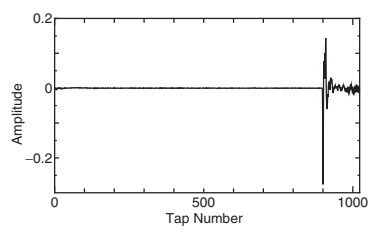

(a) $s^{11}$

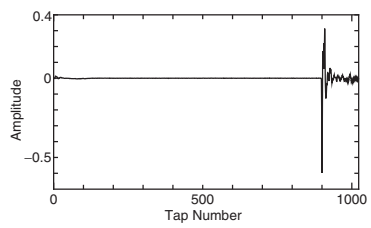

(c) $s^{21}$

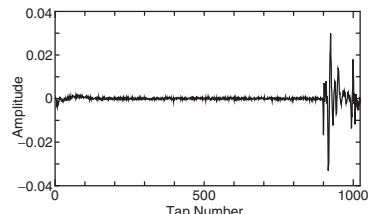

(b) $s^{12}$

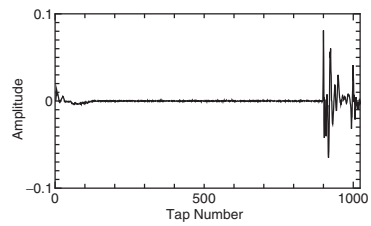

(d) $s^{22}$
Fig. 20 Coefficients of auxiliary filters $(J=896$ and $D=512)$.

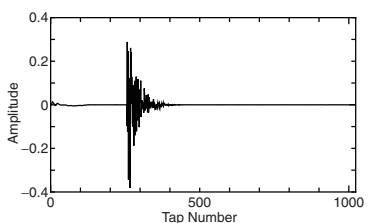

(a) $\hat{p}^{11}$

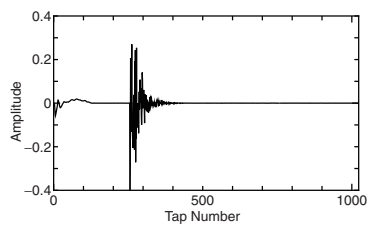

(c) $\hat{p}^{21}$

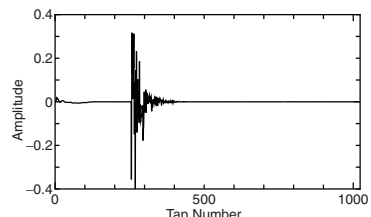

(b) $\hat{p}^{12}$

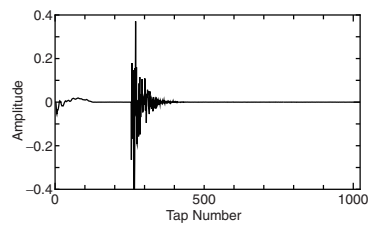

(d) $\hat{p}^{22}$
Fig. 21 Impulse responses of created sound field $(J=1,280$ and $D=256)$.

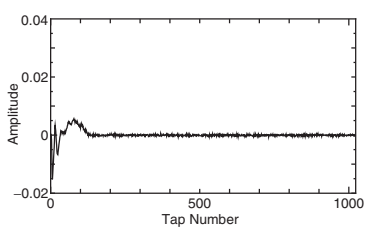

(a) $s^{11}$

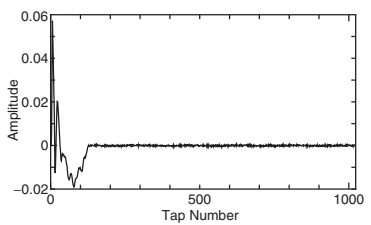

(c) $s^{21}$

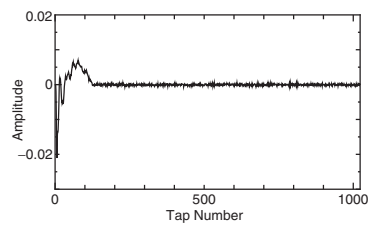

(b) $s^{12}$

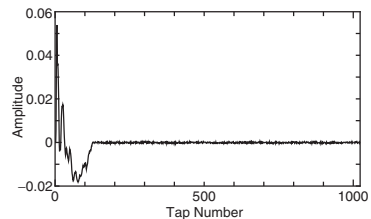

(d) $s^{22}$
Fig. 22 Coefficients of auxiliary filters $(J=1,280$ and $D=256)$.

decrease. This can also be recognized from the auxiliary filter coefficients shown in Fig. 22.

Figure 23 shows the transition of the estimation errors obtained by increasing the delays and the number of taps of the inverse filters from 256 to 768 samples and from 896 to

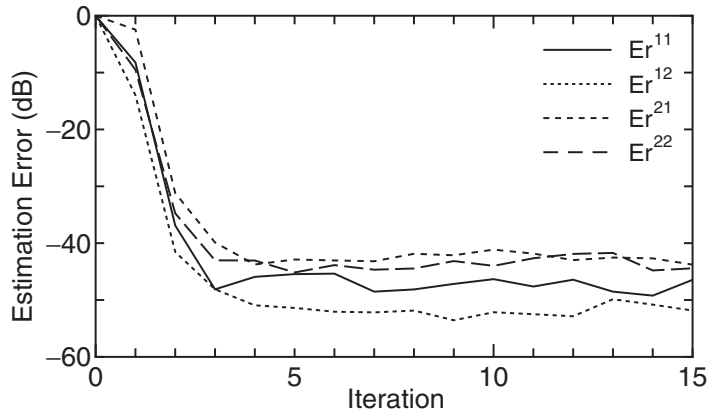

Fig. 23 Transition of estimation errors $(J=1,920, D=768)$.

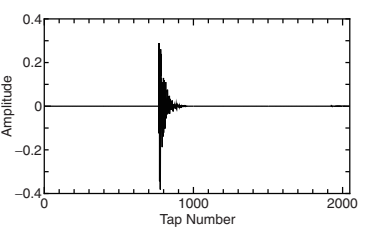

(a) $\hat{p}^{11}$

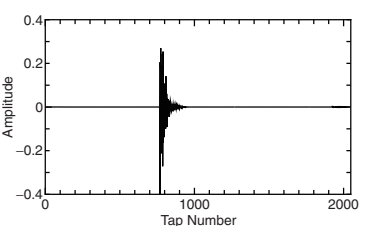

(c) $\hat{p}^{21}$

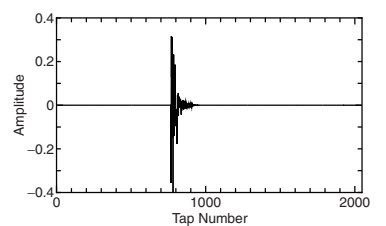

(b) $\hat{p}^{12}$

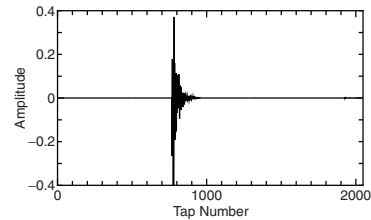

(d) $\hat{p}^{22}$
Fig. 24 Impulse responses of created sound field $(J=1,920$ and $D=768)$.

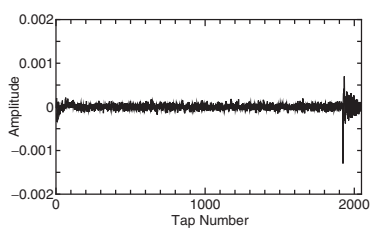

(a) $s^{11}$

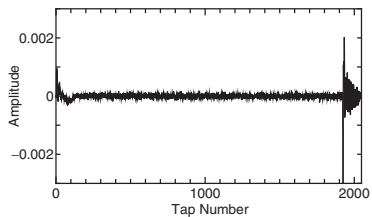

(c) $s^{21}$

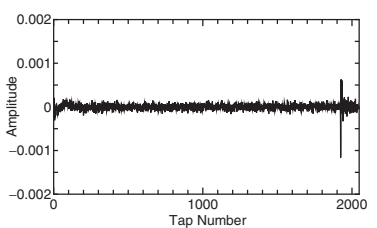

(b) $s^{12}$

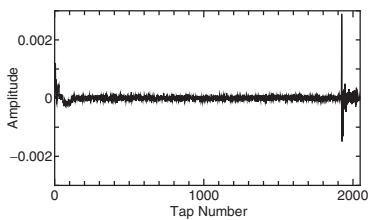

(d) $s^{22}$
Fig. 25 Coefficients of auxiliary filters $(J=1,920$ and $D=768)$.

1920 taps, respectively, where the other conditions are the same as those in the case of Fig. 16. As seen from the result, the estimation errors decrease to less than $-40 \mathrm{~dB}$, which demonstrates that the delays and the number of taps are sufficient. Figures 24 and 25 show the impulse responses of the created sound field and the coefficients of the auxiliary filters, respectively. In these results, we see negligible preceding echoes and tail echoes in the impulse responses. 
The above results indicate that the proposed method can separately identify the insufficiency of the number of ttaps and added delays through the coefficients of the auxiliary filters. We can thereby adjust them properly.

\section{CONCLUSION}

In this paper, we proposed a sound field creation system based on the simultaneous equations method and verified that the proposed system successfully creates the target sound field. In addition, we showed that the insufficiency of the delays to be added to the target sound field filters and of the number of taps given to the inverse filters can be individually identified by monitoring the coefficients of the auxiliary filters. This means that the proposed method can properly adjust the amount of added delays and the number of taps.

In the future work, we will apply the proposed principle to experimental systems, and verify that the proposed system works in practice.

\section{REFERENCES}

[1] J. Bauck and D. H. Cooper, "Generalized transaural stereo and applications," J. Audio Eng. Soc., 44, 683-705 (1996).

[2] P. A. Nelson, H. Hamada and S. J. Elliott, "Inverse filters for multi-channel sound reproduction," IEICE Trans. Fundam., E75-A, 1468-1473 (1992).

[3] T. Tokuno, O. Kirkeby, P. A. Nelson and H. Hamada, "Inverse filter of sound reproduction system using regularization," IEICE Trans. Fundam., E80-A, 809-820 (1997).

[4] H. Nakajima, M. Miyoshi and M. Tohyama, "Sound field control by indefinite MINT filters," IEICE Trans. Fundam., E80-A, 821-824 (1997).

[5] A. Kaminuma, S. Ise and K. Shikano, "A method of an inverse filter design for multi-channel sound reproduction system using minimum-norm-solution in frequency domain," J. Acoust. Soc. Jpn. (J), 57, 175-183 (2001) (in Japanese).

[6] Y. Furumi, H. Hokari and S. Shimada, "A study on loudspeaker arrangement in multi-channel transaural system for sound image reproduction," IEICE Trans. Fundam., J83-A, 920-923 (2000) (in Japanese).

[7] Y. Takekura, H. Saruwatari and K. Shikano, "Sound reproduction system including adaptive compensation of temperature fluctuation effect for broad-band sound control," IEICE Trans. Fundam., E85-A, 1851-1860 (2002).

[8] T. Tazaki, K. Tukamoto, Y. Kajikawa and Y. Nomura, "A study on sound reproduction system using the perturbation method," IEICE Tech. Rep., EA2005-8, pp. 13-18 (2005) (in Japanese).

[9] T. Horiuchi, H. Hokari, S. Shimada and T. Inada, "Adaptive estimation of transfer function for sound localization using stereo earphone-microphone combination," IEICE Trans. Fundam., E85-A, 1841-1850 (2002).

[10] K. Fujii, K. Yamaguchi, S. Hashimoto, Y. Fujita and M. Muneyasu, "Verification of simultaneous equations method by an experimental active noise control system," Acoustical Science \& Tech., 27, 270-277 (2006).

[11] M. M. Sondhi, D. R. Morgan and J. L. Hall, "Stereo-phonic acoustic echo cancellation-An overview of the fundamental problem," IEEE Signal Process. Lett., 2, 148-150 (1995).

Tadashi Ujino received B.E. and M.E. degrees in electrical engineering from University of Hyogo in 2006 and 2008, respectively. He joined FUJUTSU TEN LTD., Kobe, Japan.

Keiichiroh Tanaka received B.E degree in acoustic design engineering from Kyushu University. He joined FUSJITSU TEN LTD., Kobe, Japan, in 2006, where he has been researching and developing acoustic signal processing systems.

Isao Wakabayashi received B.E and M.E degrees in science from Osaka University. He joined FUJITSU TEN LTD., Kobe, Japan, in 2005, where he has been researching and developing acoustic signal processing systems.

Kensaku Fujii received B.E., M.E. and Dr. E. degrees in electrical engineering from Osaka Prefecture University in 1970, 1972 and 1992, respectively. He joined FUJITSU LTD., Kawasaki, Japan, in 1972, where he first engaged in the design of electric telephone exchanger. From 1989 to 2001, he studied the application of digital signal processing to echo canceller and active noise control systems. Since 2001, he has been with Himeji Institute of Technology (currently University of Hyogo), Himeji, Japan, where he is a professor in the Graduate School of Electrical Engineering. His research interests are in the area of digital signal processing and its application. He received the 1993 Best paper Award from the IEICE. From 1994 to 1998, he was an Associate Editor of Trans. on Fundamentals of IEICE. He is a member of the IEEE and the Acoustical Society of Japan.

Mitsuji Muneyasu received the B.E. and M.E. degrees in system engineering from Kobe University in 1982 and 1984, respectively, and Doctor of Engineering from Hiroshima University, Japan, in 1993. In 1984, he joined Oki Electric Industry Co., Ltd., in Tokyo, Japan. From 1990 to 1991, he was a Research Assistant at the Faculty of Engineering, Tottori University, Tottori, Japan. From 1991 to 2001, he was a Research Assistant and Associate Professor at the Faculty of Engineering, Hiroshima University, Higashi-Hiroshima, Japan. Since 2001, he has been with the Faculty of Engineering, Kansai University, Osaka, Japan, where he is currently a Professor. His research interests include image processing theory and nonlinear digital signal processing. He is a member of IEEE and IPSJ. 